Working Paper No. 615, 2004

Equilibrium Search Unemployment with Explicit Spatial Frictions

by Etienne Wasmer and Yves Zenou

IUI, The Research Institute of Industrial Economics

P.O. Box 5501

SE-114 85 Stockholm

Sweden 


\title{
Equilibrium Search Unemployment with Explicit Spatial Frictions*
}

\author{
Etienne Wasmer ${ }^{\dagger}$ and Yves Zenou
}

\section{December 18, 2001. First Revision August 1, 2003. Second Revision March 16, 2004.}

\begin{abstract}
Assuming that job search efficiency decreases with distance to jobs, workers' location in a city depends on spatial elements such as commuting costs and land prices and on labour elements such as wages and the matching technology. In the absence of moving costs, we show that there exists a unique equilibrium in which employed and unemployed workers are perfectly segregated but move at each employment transition. We investigate the interactions between the land and the labour market equilibrium and show under which condition they are interdependent. When relocation costs become positive, a new zone appears in which both the employed and the unemployed co-exist and are not mobile. We demonstrate that the size of this area goes continuously to zero when moving costs vanish. Finally, we endogeneize search effort, show that it negatively depends on distance to jobs and that long and short-term unemployed workers coexist and locate in different areas of the city.
\end{abstract}

J.E.L. Classification: E24, J41, R14

Keywords: local labour markets, relocation costs, search effort, job matching.

\footnotetext{
${ }^{*}$ We are grateful to the editor Alison Booth and three anonymous referees for their helpful comments. Yves Zenou thanks the Marianne and Marcus Wallenberg Foundation for financial support.

${ }^{\dagger}$ UQAM, University of Metz, ECARES-ULB and CEPR. Address: University du Quebec a Montreal (UQAM), Departement des Sciences Economiques, Case Postale 8888, Succursale Centre-Ville, Montreal (Quebec), CANADA H3C 3P8. E-mail: wasmer.etienne@uqam.ca

${ }^{\ddagger}$ IUI, University of Southampton, GAINS and CEPR. Address: IUI, The Research Institute of Industrial Economics, Box 5501, 11485 Stockholm, Sweden. E-mail: yvesz@iui.se
} 


\section{Introduction}

It has been recognized for a long time that distance interacts with the diffusion of information. In his seminal contribution to search, Stigler (1961) puts geographical dispersion as one of the four immediate determinants of price ignorance. The reason is simply that distance affects various costs associated with search. In most search models, say for example Diamond (1981 and 1982), distance between agents or units implies a fixed cost of making another draw in the distribution. In other words, a spatial dispersion of agents creates more frictions and thus more unemployment. Conventional labour economics faces difficulties in thinking about these spatial differences because it is biased towards the notion of a spaceless marketplace ruled by the walrasian auctioneer.

This is a weakness of the analysis since empirical evidence supports the idea of a clear spatial dimension of labour markets (see for example the literature survey by Crampton, 1999). There are in fact several channels through which space affects the labour market. First, workers who live further away from jobs may have poorer labour market information and be less productive than those living closer to jobs (Seater, 1979). This is particularly true for younger and/or less-skilled workers who rely heavily on informal search methods for obtaining employment (Holzer, 1987). ${ }^{1}$ The reliance on these informal methods of job search suggests that information on available job opportunities may decay rapidly with the distance from home (Ihlanfeldt and Sjoquist, 1990). Second, distance also implies higher commuting costs for the unemployed, which directly affect the search process (Van Ommeren et al., 1997). Third, workers residing too far away from jobs may quit their job more frequently because of too long commuting distances (Zax and Kain, 1996). Finally, employers may discriminate against applicants living in remote areas because of lower productivity (Zenou, 2002). As a result it is commonly observed that unemployment rates differ strongly across as well as within local labour markets (see e.g. Blanchflower and Oswald, 1994, Martson, 1985, Topa, 2001).

The interaction between space and labour markets is thus complex. We have divided our research questions into two parts. In a companion paper (Wasmer and Zenou, 2002), the

\footnotetext{
${ }^{1}$ In Holzer (1988), it is shown that among 16-23 years old workers who reported job acceptance, $66 \%$ used informal search channels (30\% direct application without referral and $36 \%$ friends/relatives), while only $11 \%$ using state agencies and $10 \%$ newspapers.
} 
focus was mainly urban and we have explicitly studied all possible urban configurations in a job-matching framework. We have in particular shown how a public transportation policy strongly depends on which type of urban equilibrium prevails.

The aim of the present paper is to focus instead on the labour market aspects of urban equilibria. To this purpose, we focus on the most relevant urban equilibria of Wasmer and Zenou (2002), the one in which the unemployed reside far away from jobs. Within this urban equilibrium, we systematically explore the role of space, and notably the spatial dimension of search. We have more specifically three questions in mind: Does search equilibrium strongly depend on these spatial terms? Do relocation costs strongly affect the equilibrium? Is longterm unemployment a phenomenon interacting with space? Our answers are yes to three questions.

In our approach, the matching of jobs and workers is a time-consuming process and the number of matches per unit of time between workers and open vacancies is represented by an aggregate matching function (à la Diamond-Mortensen-Pissarides). Even if firms pay workers their reservation wage, there is still some unemployment in the area (due to stochastic rationing not being eliminated by price adjustment). However, in this line of search models, the spatial dimension is often implicit. Here, we explicitly introduce it by considering that the distance between workers' residential locations and jobs plays an adverse role in the formation of matches. In this respect, our model can be viewed as a natural extension of the standard matching model. The land market will be kept rather simple in order to provide closed-form solutions. We consider a closed piece of land (that can be thought as an urban area, a city, an agglomeration or a region). This area is monocentric, i.e., firms are exogenously located in an employment center and workers consume inelastically one unit of space. In our analysis, local factors (rental price, distance to the employment center) and global factors (labour market tightness, wages) influence workers' location decisions, i.e. the land market equilibrium. Within this framework, we can have different land market equilibria. We only focus here on the equilibrium in which the unemployed reside further away from the employment center.

We first study the case of zero-relocation costs so that workers change location as soon as they change employment status. We derive the labour market equilibrium in which spatial unemployment is due to frictions in the labour market. On the one hand, the land market equilibrium depends on aggregate variables (such as wages and labour market tightness) since these variables affect location choices of workers. On the other hand, the labour market 
equilibrium crucially depends on the land market equilibrium configuration. Indeed, the efficiency of aggregate matching depends on the average location of the unemployed. We first show that there exists a unique and stable market equilibrium in which both land and labour markets are solved for simultaneously. We then show that space has an important role on the interaction between land and labour markets. We notably decompose the equilibrium unemployment rate into two parts: a pure non-spatial one (which corresponds to the standard matching model) and a mixed of non-spatial and spatial elements, the first element amplifying the other one. In other words, space adds to search frictions in the labour market by making the access to jobs more difficult.

We then investigate the case of positive moving costs paid when agents relocate within the city. $^{2}$ Between the two perfectly segregated areas appears a zone where both the employed and the unemployed co-exist and are not mobile. We show that the size of this area goes continuously to zero when moving costs vanish.

We finally study the endogeneity of job search effort. We show that long and short-term unemployed workers emerge and locate in different parts of the city.

The remainder of the paper is organized as follows. The next section presents the model with no relocation costs and shows the different roles of space in the determination of equilibrium unemployment. Section 3 analyzes the role of positive relocation costs. Section 4 analyzes the case of endogenous search effort. Finally, section 5 concludes.

\section{Benchmark model}

\subsection{The setup}

Firms and workers are all (ex ante) identical and the labour force is normalized to 1 . It follows that the unemployment rate $u$ is equal to the unemployment level. Throughout the paper, we only focus on aggregate steady-state equilibria. A firm can be in two distinct states: either associated with a worker whose production is $y$ units of output or unproductive in absence of a suitable worker. To find that suitable worker, the firm posts a vacancy. The rate per unit of time at which the vacancy is filled is constant over time, i.e. follows a random Poisson process. Symmetrically, workers searching for a job will meet a vacancy at a constant rate according to another Poisson process. The two processes are governed according to a matching function determining the total number of contacts per unit of time: $h(\bar{s} u, V)$, where

\footnotetext{
${ }^{2}$ See Haavio and Kauppi (2003) for a detailed two-region analysis for a similar focus with in addition credit market imperfections.
} 
$V$ denotes the number of vacancies. Observe that this matching function is written under the assumption that all firms are located in one fixed location. This is the spatial structure of cities that we assume throughout this paper. Individual workers $i$ have heterogenous search efficiencies equal to $s_{i}$. Accordingly, the notation $\bar{s}$ represents the average efficiency of search of the unemployed workers.

As usual, $h($.$) is assumed to be increasing both in its arguments, concave and exhibits$ constant returns to scale. The probability for a vacancy to be filled per unit of time is then $\frac{h(\bar{s} u, V)}{V}$, and using the constant return to scale assumption, it can be written as $h\left(\frac{1}{\theta}, 1\right) \equiv q(\theta)$ where $\theta=V / u \bar{s}$ is a measure of labour market tightness in efficiency units and $q(\theta)$ is a Poisson intensity. Similarly, for a worker $i$ with efficiency $s_{i}$, the probability of obtaining a job per unit of time is: $\frac{h(\bar{s} u, V)}{u} \frac{s_{i}}{\bar{s}} \equiv \theta q(\theta) s_{i} \equiv p_{i}$, where $p_{i}$ is defined as the intensity of the exit rate from unemployment. The deviation from the standard model of job matching (Mortensen and Pissarides, 1999, Pissarides, 2000) where there is no spatial dimension is the following: the search efficiency parameter $s_{i}$ depends on the location of the unemployed workers in the city. It is further assumed that the closer the residential location to the workplace, the better the efficiency and the more likely is a contact: $s_{i}=s\left(d_{i}\right)$, where $d_{i}$ is the location of the worker with $\left.s^{\prime}\left(d_{i}\right)<0\right) .{ }^{3}$ For analytical simplicity, we assume that:

$$
s_{i}\left(d_{i}\right)=s_{0}-a d_{i}
$$

with $s_{0}>0$ and $a>0$. In this formulation, $s_{0}$ denotes the outcome of search effort (such as writing letters, buying newspapers ... ) in absence of spatial interaction in search while $a$ represents the loss of information per unit of distance.

Once the match is made, the wage is determined by a generalized Nash bargaining solution specified later on. There is also a probability $\delta$ per unit of time that the match is destroyed. In order to determine the (general) equilibrium, we will proceed as follows. We first study the partial land market equilibrium configuration. Then, depending on the location of workers and thus on the aggregate search efficiency $\bar{s}$, we determine the partial labour market equilibrium. Hereafter, by labour (respectively land market) equilibrium, it has to be understood partial equilibrium. The general equilibrium involves two markets, and will be denominated a 'market equilibrium'.

By denoting by $R(d)$ the land market price at a distance $d$ from the city-center, by $w$ the

\footnotetext{
${ }^{3}$ In section 4 , we relax this assumption and show that $s_{i}$ and $d_{i}$ are negatively related when search effort is affected by commuting costs.
} 
wage earned by workers and by $u$ the unemployment rate, we have the following definition. A market equilibrium is a 4-uple $(R(d), w, \theta, u)$ that is solved simultaneously for the land use equilibrium and the labour market equilibrium. We shall give below more precise definitions of these two markets.

\subsection{The allocation of workers in space}

We assume, as it is quite standard in urban economics (see e.g. Brueckner, 1987 or Fujita, 1989), that the area is monocentric, i.e., all firms are exogenously located in the employment center. The area is also linear, closed and landlords are absent. The center of the city is located in $d=0$ where jobs are also located. Workers are uniformly distributed in space, and decide about their optimal residence between the employment center and the city-fringe. All consume inelastically one unit of land.

All workers incur a travel cost to the center. Let us denote by $\tau_{e} d$ and $\tau_{u} d$ the transportation cost at a distance $d$ from the employment center for respectively working activities and unemployed specific activities (interviews, registration), with $\tau_{e}>\tau_{u}>0$. This is in line with studies showing that time spent to job search activities is marginal compared to employment time (see for instance Layard, Nickell and Jackman, 1991). They also pay the land rent costs at the market price $R(d)$, and receive a wage $w$ when employed and unemployment benefits $b$ when unemployed.

We denote by $U(d)$ and $W(d)$ the expected discounted lifetime net income of the unemployed and the employed respectively when they locate at distance $d$ from jobs. We assume that location changes are costless ${ }^{4}$ and further assume perfect financial markets. With the Poisson probabilities defined above, infinitely-lived workers have then the following intertemporal utility functions $U^{*}=\underset{d}{\operatorname{Max}} U(d)$ and $W^{*}=\underset{d}{\operatorname{Max}} W(d)$ defined recursively through the following Bellman equations:

$$
\begin{gathered}
r U^{*}=b-\tau_{u} d^{* u}-R\left(d^{* u}\right)+p\left(d^{* u}\right)\left[W\left(d^{* w}\right)-U^{*}\right] \\
r W^{*}=w-\tau_{e} d^{* w}-R\left(d^{* w}\right)+\delta\left[U\left(d^{* u}\right)-W^{*}\right]
\end{gathered}
$$

where $r$ is the exogenous discount rate and where $d^{* u}\left(\right.$ resp. $\left.d^{* w}\right)$ means that $d^{* u}$ is chosen so as to maximize $U$ (resp. $W$ ) . Let us comment (2). When a worker is unemployed today,

\footnotetext{
${ }^{4}$ This is a simplifying assumption. It implies that workers change location as soon as they change employment status. In the context of European labour markets in which workers tend to experience long unemployment spells, it may be a rather good approximation since, when workers become unemployed, they will be less able to pay land rents and, after some time, they will have to relocate in cheaper places. We however relax this assumpion in section 3 .
} 
he/she resides in $d^{*}$ and his/her net income is $b-\tau_{u} d^{* u}-R\left(d^{* u}\right)$. Then, he/she can get a job with a probability $p\left(d^{* u}\right)$ and if so, he/she relocates optimally in $d^{* w}$ and obtains an increase in income of $W^{*}-U^{*}$. The interpretation of (3) is similar.

It is important to notice that, thanks to the assumption of no relocation costs, the equilibrium is such that all the unemployed enjoy the same level of utility $U=\bar{U}=U^{*}$. This is also true for the employed $W=\bar{W}=W^{*}$. Indeed, any utility differential within the area would lead to the relocation of some workers up to the point where differences in utility disappear.

In order to solve the workers' location problem, let us introduce the concept of bid rents (Fujita, 1989). They are defined as the maximum land rent at a distance $d$ that each type of worker is ready to pay in order to reach his/her respective equilibrium utility level. See Appendix 1 for an expression for these bid rents from equations (2) and (3). The land use equilibrium $R(d)$ is then the upper envelop of all workers' bid rents and of the constant rent outside the city $R_{A}$. Formally, $R(d)=\max \left\{\Psi_{u}(d, \bar{U}, \bar{W}), \Psi_{e}(d, \bar{U}, \bar{W}), R_{A}\right\}$, at each $d \in[0,1])$. Because $p^{\prime \prime}(d)=0$, all bid rents are linear and their slopes are given by:

$$
\begin{aligned}
& \frac{\partial \Psi_{u}(d, \bar{U}, \bar{W})}{\partial d}=-\tau_{u}+p^{\prime}(d)(\bar{W}-\bar{U})<0 \\
& \frac{\partial \Psi_{e}(d, \bar{U}, \bar{W})}{\partial d}=-\tau_{e}<0
\end{aligned}
$$

where $p^{\prime}(d)=\theta q(\theta) s^{\prime}(d)<0$. The absolute value of the slopes are the marginal cost that a worker is ready to pay in order to be marginally closer to the employment center by one unit of space. Depending on the relative slopes, only two land market equilibria are possible: either the employed reside at the vicinity of the employment center and the unemployed at the outskirts of the area or we have the reverse pattern. In this paper, we only focus on the equilibrium where the unemployed are far away from jobs ${ }^{5}$ because we want to show how the spatial access to jobs matters for the labour market outcomes of workers. The condition for this equilibrium to occur is that the slope of the bid rent of the employed exceeds that of the unemployed, that is:

$$
\tau_{e}-\tau_{u}>\theta q(\theta) a(\bar{W}-\bar{U})
$$

This is quite intuitive: for the employed to occupy the core of the area, it must be that their bid rents exceed those of the unemployed. This condition states that the differential in commuting costs between the employed and the unemployed must be higher than the

\footnotetext{
${ }^{5}$ In Wasmer and Zenou (2002), we investigate both urban equilibria.
} 


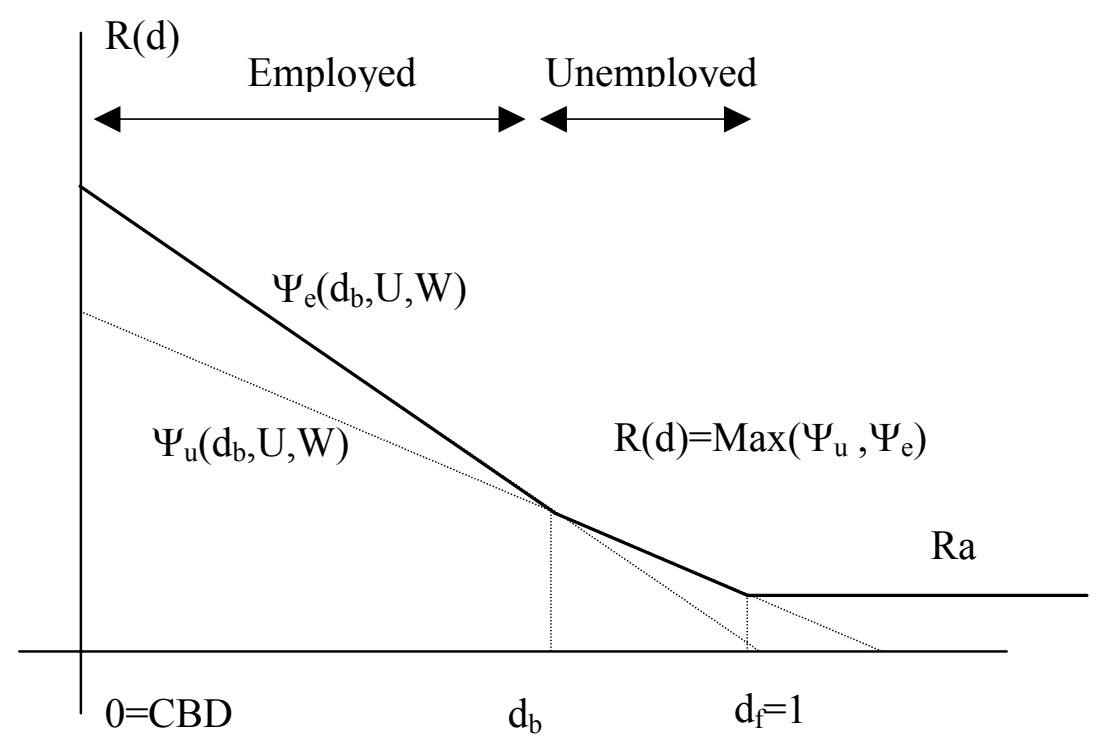

Figure 1: Bid rents and equilibrium rent, location of workers within a city.

expected return for the unemployed of being more efficient in search by being marginally closer to the center.

In this context, the land market equilibrium $\left(\bar{U}, \bar{W}, d_{b}\right)$ is such that:

$$
\begin{aligned}
d_{b} & =1-u \\
\Psi_{e}\left(d_{b}, \bar{U}, \bar{W}\right) & =\Psi_{u}\left(d_{b}, \bar{U}, \bar{W}\right) \\
\Psi_{u}\left(d_{f}, \bar{U}, \bar{W}\right) & =R_{A}=0
\end{aligned}
$$

This equilibrium is illustrated in Figure 1. The average efficiency intensity is equal to:

$$
\bar{s}=s_{0}-a \bar{d}=s_{0}-a\left(1-\frac{u}{2}\right)
$$

Thus, $\bar{s}$ is increased by a constant part $s_{0}$, and is decreased by a higher distance from the center of the average unemployed worker. At a constant unemployment rate, it decreases with the gradient parameter $a$.

\subsection{The labour market}

Given our land market equilibrium, we can now define the labour market equilibrium and then, solve the general problem. Let us first have the following definition of a labour market equilibrium: A (steady-state) labour market equilibrium $(w, \theta, u)$ is such that, given the 
matching technology defined by $h(\bar{s} u, V)$, all agents (workers and firms) maximize their respective objective function, i.e. this triple is determined by a free-entry condition for firms, a wage-setting mechanism and a steady-state condition.

The first part of the labour block comes from a free-entry condition for firms, leading to the determination of labour demand. As in standard search analysis (Pissarides, 2000), it is given by the following decreasing relation between labour market tightness and wages:

$$
J=\frac{\gamma}{q(\theta)}=\frac{y-w}{r+\delta}
$$

where $\gamma$ and $y$ are respectively the cost of holding a vacant job and worker's productivity. Indeed, the value of a job $J$ must in equilibrium be equal to the expected search cost, i.e. the cost per unit of time multiplied by the average duration of search for the firm.

Let us now determine the wage. We do not allow firms to offer different wages according to residential location. This is a legal constraint based on the fact that, in the real world, one never observes firms that discriminate across identical workers according to their place of residence. As a result, we consider here only equilibria in which wages are constant in space. ${ }^{6}$

Each period, the total surplus is shared between firms and workers through a bargaining according to the generalized Nash-sharing rule. Let us denote by $0 \leq \alpha \leq 1$ the share of workers in the total intertemporal surplus $\bar{W}-\bar{U}+J$. Then, solving the Nash-bargaining problem, it is easy to show (see Appendix 1) that

$$
w=(1-\alpha)\left[b+\left(\tau_{e}-\tau_{u}\right) d_{b}\right]+\alpha\left[y+\left(s_{0}-a d_{b}\right) \theta \gamma\right]
$$

where $d_{b}=1-u$. We thus have an expression for wages that combines usual non-spatial effects with additional spatial effects. The former are given by $(1-\alpha) b+\alpha\left[y+s_{0} \theta \gamma\right]$ and correspond to the usual Nash-bargaining solution in Pissarides (2000). The additional (spatial) part contains first $(1-\alpha)\left(\tau_{e}-\tau_{u}\right) d_{b}$, i.e. a pure spatial term: this is what firms must pay to share the costs incurred by the workers. These costs appear as the difference between the employed and the unemployed of the cost of the marginal worker who is the furthest away from the employment center, i.e. located at $d_{b}=1-u$ (at the point in space where the land-rent difference between an employed and an unemployed agent vanishes). ${ }^{7}$ The second

\footnotetext{
${ }^{6}$ It is possible that another equilibrium exists, in which wages would depend on distance even though the intertemporal utility of the employed $\bar{W}$ remains constant over space. In this case, the employed's land rent would adjust in order for $\bar{W}$ to stay constant. There is no clear argument to rule this equilibrium out and we thank a referee for pointing it out. However, this equilibrium is quite unlikely since, to be sustained, it requires a lot of coordination between firms and workers.

${ }^{7}$ See Smith and Zenou (1997) for a similar effect in an efficiency wage framework.
} 
part of the additional term is $-\alpha a d_{b} \theta \gamma$. It involves both spatial and labour elements. Indeed, when $d_{b}$ increases, the unemployed worker who is the closest from jobs (the one situated at $d_{b}=1-u$ ) is even less close to jobs (spatial element) and thus has a lower search efficiency (labour element). The outside option of employed workers then decreases, which implies a reduction in wages.

\subsection{The steady-state equilibrium}

Using $w$ and $\theta$, the condition (6) that ensures that the land market equilibrium always exists and is unique can be rewritten as: ${ }^{8}$

$$
\theta<\bar{l}=\frac{1-\alpha}{\alpha} \frac{\tau_{e}-\tau_{u}}{\gamma a}
$$

The intuition of this condition is straightforward: when the difference in commuting costs, $\tau_{e}-\tau_{u}$, is large and/or when the loss of information per unit of distance, $a$, is small and/or the search cost of firms, $\gamma$, is small, and/or the workers' bargaining strength, $\alpha$, is low, then the employed bid away the unemployed at the periphery of the city.

Combining (11) and (12), one can eliminate the wage and obtain a relation between $\theta$ and $u$ as follows (recalling that $d_{b}=1-u$ ):

$$
y-b=\frac{\gamma}{q(\theta)}\left[\frac{\delta+r+\theta q(\theta) s\left(d_{b}\right) \alpha}{1-\alpha}\right]+\left(\tau_{e}-\tau_{u}\right) d_{b}
$$

We can now close the model by providing another relation between $\theta$ and $u$. This relation is given by the existence of a steady-state condition on flows:

$$
\theta q(\theta) \bar{s} u=(1-u) \delta
$$

Proposition 1 When the parameters are such that $\theta^{*}<\bar{l}$, then, there exists a unique market equilibrium $\left(R(d), \theta^{*}, u^{*}, w^{*}\right)$ in which the unemployed reside far away from jobs and the employed close to jobs.

Proof. See Wasmer and Zenou (2002).

\subsection{The role of space in the theory of unemployment}

\subsubsection{Interaction between land and labour markets}

The interaction between land and labour markets is partly due to the dependence of search efficiency on distance. To show that, we proceed a contrario: we assume first that wages are

\footnotetext{
${ }^{8}$ Using (11) and the bargaining equation in Appendix 1, it is easy to verify that condition (6) can be written as (13).
} 
exogenous and $a=0$. In this case, both markets are independent. When we relax exogenous wages and keep $a=0$, there is a one-way interaction between markets: the labour market does not depend on the land market but the land market equilibrium depends on the labour market equilibrium through labor market tightness as workers locate in one configuration or the other depending notably on $\theta^{*}$.

Finally, as soon as $a>0$, one has a general equilibrium interaction between the markets. Indeed, one of the key assumption of our model is that the search efficiency $s_{i}$ of each worker $i$ depends on the distance between residence and the job-center, i.e., $s_{i}=s_{i}(d)$ with $s_{i}^{\prime}(d)<0$. This implies that the land and labour markets are interdependent. Indeed, on the one hand, the labour market strongly depends on the land market since the equilibrium values of $u^{*}, V^{*}$ and $\theta^{*}$ are affected by the value of $\bar{s}$. On the other hand, the land market strongly depends on the labour market since the inequality (6) determining the land market equilibrium configuration, $\theta^{*}<\bar{l}$, depends on the value of $\theta^{*}$.

To evaluate the implications of this relation $s_{i}(d)$, let us assume that $s_{i}$ is independent of $d$ ( $a=0$ so that $\bar{s}=s_{0}$ ) but workers still locate in the city and thus bear land rents and commuting costs. In this context, the inequality (6) reduces to $t_{e}-t_{u}>0$. In other words, the land market equilibrium is independent of the labour market equilibrium. The location choices of the employed and the unemployed, which depend on the slopes of the bid rents, involve only transportation costs. So, since $t_{u}<t_{e}$, the unemployed reside at the outskirts of the city, irrespective of the labour market equilibrium outcome. When wages are exogenous, we do not have anymore equation (12) but instead $w=\bar{w}$. So, the equilibrium is defined by two equations, (11) and (15) in which $w=\bar{w}$. Therefore, when wages are exogenous, the equilibrium unemployment and vacancy rates would be exactly the same as in the standard non-spatial matching models (see e.g. Pissarides, 2000) and $\bar{s}=s_{0}$ is independent of the land use equilibrium. On the contrary, when the wage is a result of a bargaining between workers and firms, the main difference with the standard non-spatial matching model is that commuting costs affect wages. We can summarize our discussion by the following table.

Table 1: Interaction between land and labour markets

\begin{tabular}{|c|c|c|}
\hline & Exogenous wages & Endogenous wages \\
\hline$a=0$ & No Interaction & Partial Interaction $(L M E \rightarrow L E)$ \\
\hline$a>0$ & Complete Interaction & Complete Interaction \\
\hline
\end{tabular}

$(L M E \rightarrow L E$ means that the interaction is from the 
land market equilibrium to the labour equilibrium)

\subsubsection{A spatial Beveridge curve}

One can further develop the specific role of space in observing that the locus of steady-state depends on $a$ and on spatial terms. For that, we describe now an intuitive way of representing the equilibrium: in the $u-V$ space.

As stated above, equation (11) determines a value of $\theta=V /(u \bar{s})$ that gives a relation between $V$ and $u$. This is an upward sloping curve in the $u-V$ space called the $V S$ curve. Furthermore, equation (15) can be rewritten as:

$$
\delta(1-u)-V q(V / u \bar{s})=0
$$

We obtain the so-called Beveridge curve $U V$. The interesting feature of this Beveridge curve is that it is indexed by $\bar{s}$, which depends on the spatial dispersion of the unemployed: a lower $\bar{s}$ is associated with an outward shift of Beveridge curve in the $u-V$ space because more vacancies are needed to maintain the steady-state level of unemployment. If $a$ increases or $s_{0}$ decreases, the Beveridge curve is shifted away from the origin meaning that the labour market is less efficient. The same would arise if the city size increased: the unemployed would be further away. We leave this 'open city case' for further research and now turn to a quantitative investigation of the role of space on labour markets.

\subsubsection{Decomposition of unemployment}

Indeed, we pursue our analysis of the importance of space in equilibrium unemployment by determining the part of unemployment only due to spatial frictions. Let us start with exogenous wages. In this case, $\theta$ is constant and determined by (11). By using (15), the unemployment rate is given by:

$$
u=\frac{\delta}{\delta+\theta q(\theta)\left[s_{0}-a(1-u / 2)\right]}
$$

Let us further define by:

$$
u_{0}=\frac{\delta}{\delta+\theta q(\theta) s_{0}}
$$

the part of unemployment that is independent of spatial frictions, i.e. when $a=0$ so that $\bar{s}_{0}=s_{0}$. By a Taylor first-order expansion for small $a / s_{0}$, we easily obtain:

$$
u^{*}=u_{0}\left[1+\frac{a}{s_{0}}\left(1-u_{0}\right)\left(1-u_{0} / 2\right)\right]=u_{0}+u^{s}
$$


where $u^{s} \equiv u_{0}\left[a\left(1-u_{0}\right)\left(1-u_{0} / 2\right) / s_{0}\right]$ is the unemployment that is only due to spatial frictions and $u_{0}$ is defined by (18). Observe that $u^{s}$ is increasing in $a / s_{0}$, the parameter representing the loss of information through distance and null when $a=0$. Observe also that the pure frictional unemployment $u_{0}$ affects $u^{s}$ in the following way:

$$
\text { If } u_{0}<1-\frac{\sqrt{3}}{3} \approx 0.42, \text { then } \frac{\partial u^{s}}{\partial u_{0}}>0
$$

In general $u_{0}<0.42$ so that $u_{0}$ affects positively $u^{s}$, showing the full interaction between land and labour markets. This is quite natural: higher 'spaceless' unemployment $u_{0}$ affects positively frictions due to spatial heterogeneity (this is a side-effect of the dispersion of space on the unemployed themselves, which increases the average distance to jobs).

Under endogenous wage setting, a larger set of parameters determines the spatial component of unemployment. First, the endogenous wage $w$ defined by (12) can be decomposed into three parts:

$$
w=w_{0}+w_{t}-w_{a}
$$

where $w_{0}=(1-\alpha) b+\alpha\left(y+s_{0} \theta \gamma\right)$ is the wage that would receive workers if all agents were located in the same point, $w_{\tau}=(1-\alpha)\left(\tau_{e}-\tau_{u}\right)(1-u)$ reflects the impact of distance on transportation costs and thus on wages, and $w_{a}=\alpha a(1-u) \theta \gamma$ the fact that search efficiency varies with distance to jobs (this was called the 'outside option effect' of distance in the previous section). By using a Taylor expansion, one could also decompose $\theta$ in different parts, thus further decomposing the spatial part of unemployment into three parts itself. This is a bit involving for just finding a decomposition looking exactly as the decomposition of wages.

Overall, compared to the non-spatial case, unemployment increases because of the loss of information due to spatial dispersion of agents and also because of the wage compensation of commuting costs. However, it also tends to decrease because of the outside option effect that reduces wages.

In addition, a natural question to raise is whether the efficiency results of the decentralized search equilibria (Lucas and Prescott 1974, Moen 1997) still holds when the spatial dispersion of agents creates frictions. We explored this question in the urban context of Wasmer and Zenou (2002, Section 5) with a focus on the subsidy on commuting costs. We notably showed that in the general case, the standard Hosios-Pissarides condition, stating that the bargaining power of workers $\alpha$ has to be equal to the (negative of) elasticity of the matching rate of 
firms $-\theta q^{\prime}(\theta) / q(\theta)$, does not hold when commuting cost between employed and unemployed differ. The reason is that the aggregate welfare function includes commuting costs and, given that employees pay higher costs, the social planner prefer a little bit more unemployment, which is obtained thanks to a higher bargaining power of workers, beyond what it takes to internalize the matching externalities.

\section{Positive relocation costs}

The model developed above had no relocation costs. Although this assumption is quite frequent in urban economics, its relevance may depend on the nature of the labour market. Indeed, when unemployment and employment spells are short (i.e. a U.S. style of labour market), it is not necessarily appealing: low-income households do not necessarily change their residential location as soon as they change their employment status. However, in a European context, long spells of employment and unemployment make it more likely that relocation and labor transitions coincide, in which case our benchmark assumption of absence of mobility costs is relevant.

Surprisingly, the issue of relocation costs has been totally ignored by the urban literature. To the best of our knowledge, the only urban/labor papers that explicitly deal with relocation costs are that of Brueckner and Zenou (2003) and Coulson, Laing and Wang (2001). In both papers, there are two areas in the city: the center and suburbs. However, in the first paper, the authors use the extreme assumption that relocation costs are so high that workers never change location. In the latter, it is assumed that it is costless to commute within an area of the city but costly to commute from one area to another. Here we investigate an intermediate case in which some workers are mobile and some are not and relocation costs are positive within an area.

\subsection{Setup and definitions}

Let us thus assume that relocation costs exist, are finite, and denote by $C$ the instantaneous amount of effort and money supported by moving individuals. To simplify, we consider now that $s$ is independent of distance (i.e. $a=0$ ) and that wages are exogenous and fixed at a level $w$.

In equilibrium, there will be four groups of agents: the mobile employed and unemployed, and the immobile employed and unemployed. Employed workers are said to be mobile (resp. immobile) when, hit by a job-destruction shock, they decide to relocate to another part of 
the city (resp. stay at the same location). A similar definition of mobility can be adapted to the unemployed depending on the occurrence of a successful application to a job.

In order to keep the model tractable, we assume that these groups always form spatially homogenous communities. In other words, we only focus on equilibria in which mobile employed workers are perfectly segregated, mobile unemployed workers are perfectly segregated, and finally the immobile workers (both employed and unemployed) are integrated and randomly but evenly distributed in a part of the city. This latter part of the land market equilibrium is natural since, in the long-run, immobile workers are distributed according to an ergodic distribution of the employment/unemployment process. Furthermore, in order to be consistent with the previous analysis, we study an urban configuration in which the employed are close to the city-center and the unemployed live at the outskirts of the city. More precisely, as $d$ increases from 0 to 1 , we will have the mobile employed, the immobile employed/unemployed and the mobile unemployed workers .

Superscripts $m$ and $i$ respectively indicate mobility and immobility. Denote by $\mathcal{E}$ and $\mathcal{U}$ the subsets of $[0,1]$ in which the employed and the unemployed respectively reside, with $\mathcal{E} \cup \mathcal{U}=[0,1]$. Denote by $\mathcal{E}^{m}$ and $\mathcal{E}^{i}$ the subsets of $\mathcal{E}$ in which the employed are mobile and immobile respectively and by $\mathcal{U}^{m}$ and $\mathcal{U}^{i}$ the subsets of $\mathcal{U}$ in which the unemployed are mobile and immobile respectively. Observe that $\mathcal{E}^{i}$ is the complement of the subset $\mathcal{E}^{m}$ in $\mathcal{E}$ and $\mathcal{U}^{i}$ is the complement of the subset $\mathcal{U}^{m}$ in $\mathcal{U}$. Observe also, because of the assumption made above (groups always form spatially homogenous communities), each subset, $\mathcal{E}^{m}, \mathcal{E}^{i}, \mathcal{U}^{m}$ and $\mathcal{U}^{i}$, is connected and we have in the city $\mathcal{E}^{m} \cup \mathcal{E}^{i} \cup \mathcal{U}^{i} \cup \mathcal{U}^{m}=[0,1]$. Observe finally that, among the immobile, the employment status is not relevant because, when someone who is employed and lives in $\mathcal{E}^{i}$ loses his/her job, he/she stays in $\mathcal{E}^{i}$. The same applies for an unemployed in $\mathcal{U}^{i}$ who has found a job. As a result, we can define a subset $\mathcal{I}=\mathcal{E}^{i} \cup \mathcal{U}^{i}=\mathcal{E}^{i}=\mathcal{U}^{i}$, which includes all the immobile (employed and unemployed). Figure 2 illustrates these different sets.

As in the previous analysis, the mobile employed leave their area after a shock to become unemployed in $\mathcal{U}^{m}$ and reciprocally, the mobile unemployed leave $\mathcal{U}^{m}$ only to go to $\mathcal{E}^{m}$. As a result, in steady-state, the intertemporal utility of the employed and the unemployed has to be constant over location and we must have $U^{m}(d)=\bar{U}^{m}$ for all $d \in \mathcal{U}^{m}$ and $W^{m}(d)=\bar{W}^{m}$ for all $d \in \mathcal{E}^{m}$. We thus obtain the following arbitrage equation in which moving costs are 


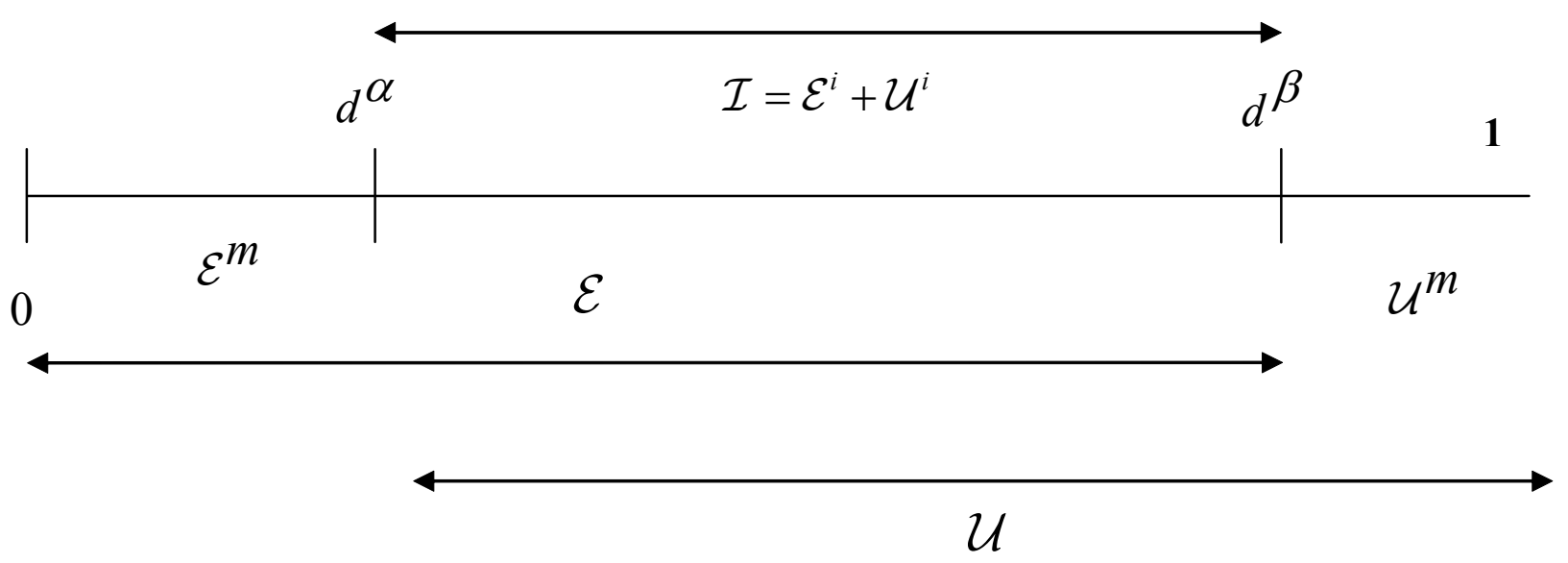

Figure 2: Location of mobile and immobile workers in the city

paid by mobile workers upon transition:

$$
\begin{aligned}
& d \in \mathcal{U}^{m} \Rightarrow r \bar{U}^{m}=b-\tau_{u} d-R(d)+p\left(-C+\bar{W}^{m}-\bar{U}^{m}\right) \\
& d \in \mathcal{E}^{m} \Rightarrow r \bar{W}^{m}=w-\tau_{e} d-R(d)+\delta\left(-C+\bar{U}^{m}-\bar{W}^{m}\right)
\end{aligned}
$$

where $p=\theta q(\theta) s_{0}$. For the immobile workers, we have instead:

$$
\begin{aligned}
& d \in \mathcal{I} \Rightarrow r U^{i}(d)=b-\tau_{u} d-R(d)+p\left[W^{i}(d)-U^{i}(d)\right] \\
& d \in \mathcal{I} \Rightarrow r W^{i}(d)=w-\tau_{e} d-R(d)+\delta\left[U^{i}(d)-W^{i}(d)\right]
\end{aligned}
$$

where, now, the intertemporal utility functions of the immobile workers explicitly depend on their location $d$.

\subsection{Equilibrium condition on location}

To characterize the new equilibrium, let us denote by $d^{\alpha}$ and $d^{\beta}$ the border between the mobile and immobile employed, and the immobile and mobile unemployed respectively. The length of $\mathcal{E}^{m}$ and $\mathcal{U}^{m}$ are thus respectively given by $d^{\alpha}$ and $1-d^{\beta}$ while the length of $\mathcal{I}$ is $d^{\beta}-d^{\alpha}$. In order to characterize this equilibrium, we must write conditions that guarantee that no immobile worker wants to relocate and that no mobile worker do not want to relocate. These conditions are twofold. A first set of conditions imposes the continuity of utility within the same employment status. Indeed, the land rent determination process imposes that the 
intertemporal utility of workers of the same employment status is continuous, i.e.

$$
\begin{gathered}
\bar{W}^{m}=W^{i}\left(d^{\alpha}\right) \\
\bar{U}^{m}=U^{i}\left(d^{\beta}\right)
\end{gathered}
$$

A second set of conditions imposes that, upon a transition (from employment to unemployment or from unemployment to employment), immobile workers do not want to relocate.

Lemma 1: For a non-degenerate equilibrium to exist, we need the two following conditions to hold:

$$
\begin{aligned}
W^{i}\left(d^{\beta}\right) & =\bar{W}^{m}-C \\
U^{i}\left(d^{\alpha}\right) & =\bar{U}^{m}-C
\end{aligned}
$$

Proof: See Appendix 2.

\subsection{Land rents}

The bid rents of workers are given in Appendix 2. We are interested in their determination around the 'frontier' points $d^{\alpha}$ and $d^{\beta}$. We show in that appendix that

$$
\begin{aligned}
& \Psi_{e}^{m}\left(d^{\alpha}, \bar{U}^{m}, \bar{W}^{m}\right)-\Psi_{e}^{i}\left(d^{\alpha}, U^{i}(d), W^{i}(d)\right)=0 \\
& \Psi_{u}^{m}\left(d^{\beta}, \bar{U}^{m}, \bar{W}^{m}\right)-\Psi_{u}^{i}\left(d^{\beta}, U^{i}(d), W^{i}(d)\right)=0
\end{aligned}
$$

or, in other words that there is no discontinuity in the equilibrium land rent at exactly $d^{\alpha}$ and $d^{\beta}$.

The equilibrium in the land market implies that the bid rents are equal to the rent, i.e.

$$
\begin{aligned}
\Psi_{e}^{i}(d) & =R(d) \text { for all } d<d^{\alpha} \\
\Psi_{e}^{i}(d) & =\Psi_{u}^{i}(d)=R(d) \text { for all } d^{\alpha}<d<d^{\beta} \\
\Psi_{u}^{i}(d) & =R(d) \text { for all } d^{\beta}<d \\
\Psi_{u}^{m}(1) & =R^{A}
\end{aligned}
$$

From the bid-rent equations in $\mathcal{U}^{m}$ and $\mathcal{E}^{m}$, we know that

$$
\begin{aligned}
& \partial R / \partial d=-\tau_{u} \text { for } d^{\beta}<d \\
& \partial R / \partial d=-\tau_{e} \text { for } d<d^{\alpha} \\
& \partial R / \partial d=-\kappa \text { for } d^{\alpha}<d<d^{\beta}
\end{aligned}
$$




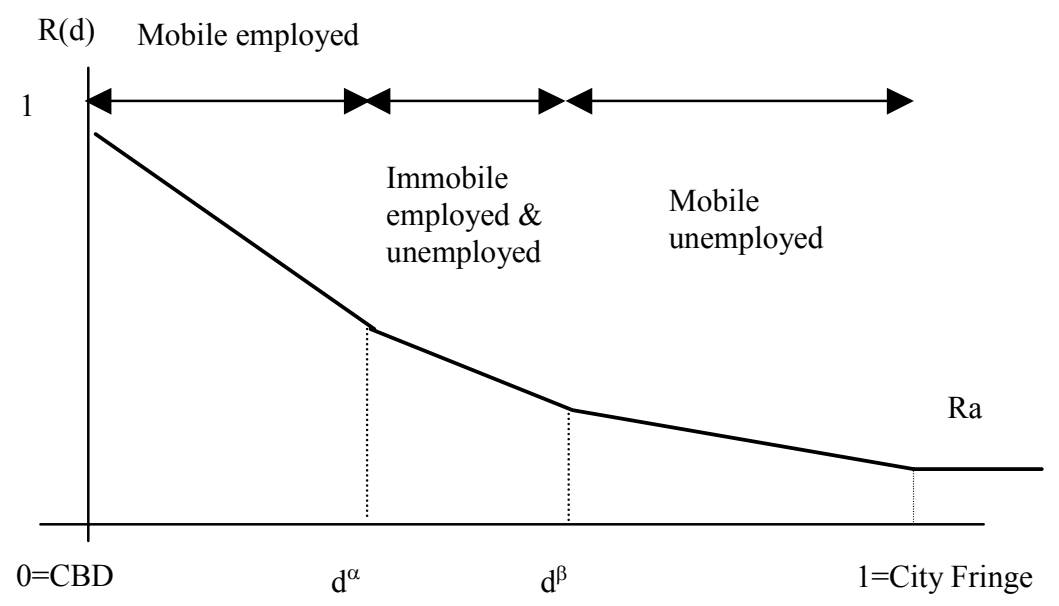

Figure 3: Profile of rents in a city with relocation costs.

where $\kappa$, the negative of the slope of the rent for mobile workers, has to be calculated. It is shown to be a constant in Appendix 2 and given by (40). By continuity, one can obtain the profile of rents that is illustrated in Figure $3 .^{9}$

\subsection{Equilibrium}

It is now time to count unknowns and equations. All rents depend on $d^{\alpha}, d^{\beta}, \bar{W}^{m}, \bar{U}^{m}$ and $U^{i}\left(d^{\alpha}\right)$ and $W\left(d^{\beta}\right)$ and finally $\partial U^{i} / \partial d$, i.e. 7 unknown variables. Equation (37) in Appendix 2 provides a link between $\bar{W}^{m}$ and $\bar{U}^{m}$. Equations (28) and (27) provide a link between $U^{i}\left(d^{\alpha}\right)$ (resp. $\left.W\left(d^{\beta}\right)\right)$ and $\bar{U}^{m}$ (resp. $\left.\bar{W}^{m}\right)$. Finally, (21) and (22) provide another link between $\bar{W}^{m}$ and $\bar{U}^{m}$, the rents being functions of the unknown described above. The last two equations are given by the continuity of utility functions, i.e. (25) and (26). All equations are linear, the system has thus one solution except in a degenerate case of measure zero in the space parameters, which we ignore hereafter.

In the absence of relocation costs, when $C$ goes to zero, we can see using (28) and (27) that $U^{i}\left(d^{\alpha}\right)=\bar{U}^{m}$ and $W^{i}\left(d^{\beta}\right)=\bar{W}^{m}$, which, using (25) and (26), leads to

$$
\bar{W}^{m}=W^{i}\left(d^{\alpha}\right)=W^{i}\left(d^{\beta}\right) \text { and } \bar{U}^{m}=U^{i}\left(d^{\alpha}\right)=U^{i}\left(d^{\beta}\right)
$$

\footnotetext{
${ }^{9}$ Given the absence of discontinuities in rents, we have $R\left(d^{\beta}\right)=\tau_{u} d^{\beta}+R_{A} ; R\left(d^{\alpha}\right)=\tau_{u} d^{\beta}+\kappa\left(d^{\beta}-d^{\alpha}\right)$ and $R(0)=\tau_{e} d^{\alpha}+\tau_{u} d^{\beta}+\kappa\left(d^{\beta}-d^{\alpha}\right)$.
} 
If we had $d^{\beta}>d^{\alpha}$, there would be a contradiction between the equations above and the fact that the surplus of immobile workers is strictly decreasing with distance as indicated in equation (38) in Appendix 2. This necessarily implies that $d^{\alpha}=d^{\beta}$. On other words, we are back to our equilibrium described in section 2 , with $a=0$ and exogenous wage.

With positive relocation costs, i.e. a strictly positive $C$, we have, using (25), (26), (28) and (27) that

$$
\begin{aligned}
& C=U^{i}\left(d^{\beta}\right)-U^{i}\left(d^{\alpha}\right) \\
& C=W^{i}\left(d^{\alpha}\right)-W^{i}\left(d^{\beta}\right)
\end{aligned}
$$

Summing those two equations and using an expression for the surplus in equation (38) in Appendix 2 leads to

$$
d^{\beta}-d^{\alpha}=\frac{2 C(r+p+\delta)}{\tau_{e}-\tau_{u}}
$$

This is a key equation determining the size of the middle area and thus the cost imposed by the full-mobility assumption made in the first section of our paper. The size of the immobility area thus increases with $C$ and with all turnover rates, and is reduced by the difference in commuting costs between the employed and the unemployed workers. The intuition is that, to remain immobile, one has to expect fast transitions in the labor market (so that waiting for another employment transition to remain in the same location is the best strategy) or low gains from mobility in terms of commuting costs.

One can also use steady-state conditions on flows of workers. The first one is related to immobile workers. Denote by $\phi$ the fraction of immobile unemployed workers in the city. Then, the unemployment rate $u$ is given by the following identity $u=\phi\left(d^{\beta}-d^{\alpha}\right)+1-d^{\beta}$. Indeed, in $\mathcal{U}^{m}$, which length is $1-d^{\beta}$, all workers are unemployed whereas among the immobile workers, there are only $\phi$ who are unemployed (we have normalized the total labor force to 1). Note that this equation brings two additional unknowns $(u$ and $\phi)$. One thus need a second steady-state condition on mobile workers:

$$
d^{\alpha} \delta=p\left(1-d^{\beta}\right)
$$

which states that, upon a $\delta$-shock, mobile employed workers relocate to the periphery, while there is a corresponding flow of mobile unemployed workers accessing employment and relocating to the center. Equations (32) and (33) uniquely determine $d^{\alpha}$ and $d^{\beta}$.

Finally, note from (40) in Appendix 2 that one can easily check that $\partial U^{i} / \partial d$ is positive (the immobile unemployed workers are better off closer to the mobile unemployed workers) 
while $\partial W^{i} / \partial d$ is negative (the immobile employed are better off close to the mobile employed workers); this justifies ex-post the assumption of Lemma 1.

To get a sense of the size of the area $\mathcal{I}$, which is equal to $d^{\beta}-d^{\alpha}$, one can make a few back-of-the-enveloppe calculations. Suppose that the cost of moving, including effort and time, is equivalent to 1000 euros. Now suppose that $\tau_{e}-\tau_{u}$ represent about 10000 euros per year (including gas, car insurance, time). Assume that $r=5 \%, \delta=2 \%$ and $p=0.5$. Thus,

$$
d^{\beta}-d^{\alpha}=\frac{2 * 1000 * 0.57}{10000}=0.11
$$

This means that eleven percent of workers in the city are immobile. Plugging into (33), we further obtain $d^{\alpha}=0.77$ and thus $d^{\beta}=0.88$, for an unemployment rate of $13 \%$. It follows that $\phi=0.1$.

In line with the mechanisms of the previous section, one can also investigate by how much the average distance of the unemployed is modified (denote it by $\bar{d}(C)$ and let us compare it to $\bar{d}(0)$ in the no-relocation cost). Some of the unemployed are immobile and have an average location of $\frac{d^{\beta}+d^{a}}{2}$. The mobile ones are located on average in $\frac{1+d^{\beta}}{2}$. The latter are a number $1-d^{\beta}$ while the former are a number $\phi\left(d^{\beta}-d^{\alpha}\right)$. Denote by $\zeta(C)=\phi\left(d^{\beta}-d^{\alpha}\right) / u$ the weight of immobile workers with $\zeta(0)=0$. Accounting for those weights, we have an average location of the employed equal to

$$
\bar{d}(C)=\zeta(C) \frac{d^{\beta}+d^{a}}{2}+(1-\zeta(C)) \frac{1+d^{\beta}}{2}
$$

The first result is that with the calculations above, we have $\bar{d}(C) \in(0.83 ; 0,94)$ where this interval is given by the average distance of the immobile and mobile workers respectively. The weight $\zeta(C)$ is equal to 0.084 which implies that $\bar{d}(C)=0.931$, while in the absence of relocation cost, the average distance would be $1-u / 2=0.935$. Again the difference is marginal and only by $0.4 \%$, although the existence of relocation costs here fixes some of the unemployed closer to jobs and avoids them being out of jobs by being far away from the employment center.

To conclude this part, one can observe that relocation costs indeed change the derivation of the equilibrium. This adds an area in the middle of the city in which employed and unemployed workers are immobile, pay the same rent and continuously overlap (there is per unit of space a density $\phi=10 \%$ ). When relocation costs disappear, we return to our previous equilibrium. This section has thus generalized the frictionless land market. 


\section{Long term unemployed and space}

Having shown the interactions between space and the labour market in assuming that search efficiency depended on space, and having extended the model to positive relocation costs, we need to better justify our initial assumption on search efficiency. We relax this assumption and instead derive this relation, in making explicit the search process and the structure of costs associated with it. We obtain new results, notably another link between the location of workers and their labour market outcomes. Notably, we introduce two classes of unemployed workers: the long run and short run unemployed workers.

For that, we go back to the model of section 2 where relocation costs were assumed to be zero. Each individual's search efficiency $s_{i}$ now depends only on his/her job search effort denoted by $e$. We assume decreasing returns to scale to effort, i.e., $s^{\prime}(e)>0$ and $s^{\prime \prime}(e) \leq 0$. As above, each interview is carried out in the employment center and thus involves transport costs. We denote by $C_{u}(e, d)$ the search costs associated with a level of effort $e$ for a worker living at a distance $d$ from the employment center. ${ }^{10}$ We assume that the search commuting cost is an increasing and convex function of the effort level $e$ devoted to job search, i.e., $\partial C_{u} / \partial e>0$ and $\partial^{2} C_{u} / \partial^{2} e \geq 0$, and that, quite naturally, $\partial^{2} C_{u} / \partial e \partial d>0$ : the search effort marginally costs more further away from jobs. There is therefore a trade-off between search costs and returns associated with a higher probability to exit from unemployment. We have therefore the following proposition.

Proposition 2 When workers choose their effort, their search efficiency and thus their probability of obtaining a job decreases with the distance to jobs.

Proof: See Appendix 3.

The intuition of this result is as follows. When choosing their optimal level of effort, the unemployed workers equalize their marginal gain (which is the probability generated by one more interview times the surplus when leaving unemployment) and their marginal loss (which is the marginal commuting cost of searching for a job). Then, because search effort marginally costs more further away from jobs, individuals search less in remote places and thus their probability to find a job decreases with distance to jobs. ${ }^{11}$

\footnotetext{
${ }^{10}$ In the previous sections, the assumption of costs was $C_{u}(e, d)=\tau_{u} d$.

11 We find no multiple equilibria in agents locations in space despite a cumulative mechanism. Indeed, being closer increases search efficiency, which further induces the incentives to be closer, etc... This is due to the assumption of decreasing returns to effort, i.e. $s^{\prime \prime}<0$. There is however a potential for multiple urban configurations if this assumption is relaxed.
} 


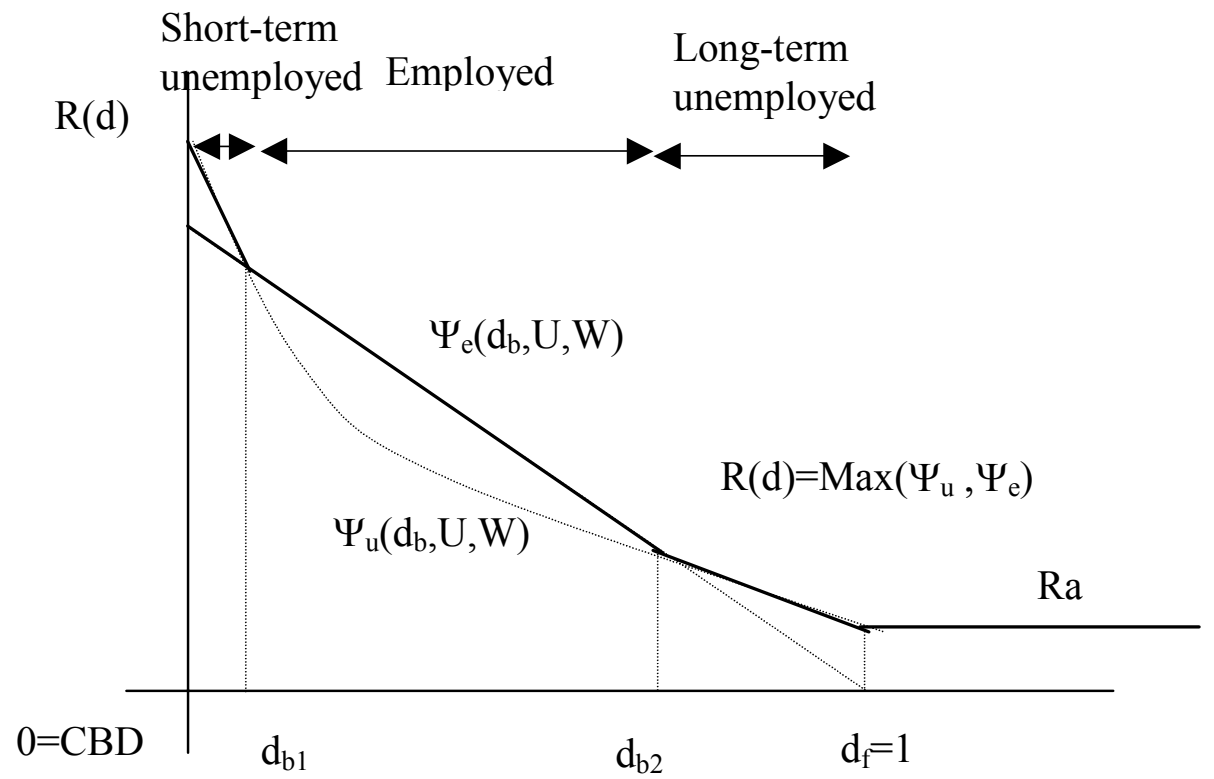

Figure 4: Location of workers within a city with endogenous search effort.

Observe that (1) is not true anymore since, according to (43) in Appendix 3, the relation between search efficiency $s($.$) and distance d$ is not linear. This implies in particular that the unemployed workers' bid rent is not anymore linear but convex. Therefore, a new land market configuration can emerge in which the unemployed reside both at the vicinity of the employment center and at the outskirts of the city while the employed live in between the unemployed (see Figure 4). ${ }^{12}$ In this case, even though all the unemployed enjoy the same utility level, the ones who reside close to the employment center experience short unemployment spells because their search efficiency is very high whereas those who live further away are long term unemployed since their probability to find a job is quite low. Indeed, either workers reside in remote areas, are long run unemployed, live on welfare but pay very low land rents or reside close to jobs, experience short unemployment spells but pay a very high land rent. Thus, space (or location) makes workers heterogeneous in terms of access to employment: those who are further away from jobs experience longer unemployment spells (see e.g. Rogers, 1997 for empirical evidence).

We can also analyze the interaction between space and equilibrium unemployment. By assuming for simplicity that wages are exogenous, the unemployment rate is given by $u=$

\footnotetext{
${ }^{12}$ See also Smith and Zenou (2003), for a similar result.
} 
$\frac{\delta}{\delta+\theta q(\theta) \bar{s}}$. Thus, the only spatial interaction in unemployment is reflected through:

$$
\bar{s}=\int_{\text {Unemployed }} s\left(e^{*}(z)\right) d z
$$

where $z$ replaces distance in the integral for notational convenience. In Appendix 3, we propose an example for an iso-elastic search-effort function.

\section{Conclusion}

In this paper, we have modelled the important interaction between the spatial dispersion of economic agents and the imperfection in information about economic opportunities. We have first demonstrated that there exists a unique and stable market equilibrium in which both land and labour markets are solved for simultaneously. We have investigated how space affects search by focusing on the interaction between land and labour markets. We have explored the mechanics of causality from the labour market to the land market and reciprocally, and further decomposed unemployment into a spatial part and a spaceless part. We have also shown the importance of relocation costs since it introduces a new area in the city where employed and unemployed workers are immobile. Finally, we have seen that when distance and search effort are complement in the cost function of individuals, long-term and short-term unemployed endogenously emerge and locate in very different location within a city.

\section{References}

[1] Blanchflower, D.G., Oswald, A.J., 1994. The Wage Curve. MIT Press, Cambridge, MA.

[2] Brueckner, J. K., 1987. The structure of urban equilibria: a unified treatment of the Muth-Mills model. In: Mills, E.S. (Ed.), Handbook of Regional and Urban Economics, Vol. 2. Elsevier Science B.V., Amsterdam, pp. 821-845.

[3] Brueckner, J.K., Zenou, Y., 2003. Space and unemployment: The labor-market effects of spatial mismatch. Journal of Labor Economics 21, 242-266.

[4] Coulson, E., Laing, D., Wang, P., 2001. Spatial mismatch in search equilibrium. Journal of Labor Economics 19, 949-972.

[5] Crampton, G.R., 1999. Urban labour markets. In: Mills, E.S., Cheshire, P. (Eds.), Handbook of Regional and Urban Economics. Vol.3. Elsevier Science B.V., Amsterdam, pp. 1499-1557.

[6] Diamond P., 1981. Mobility costs, frictional unemployment, and efficiency. Journal of Political Economy 89, 798-812. 
[7] Diamond P., 1982. Aggregate demand management in search equilibrium. Journal of Political Economy 89, 798-812.

[8] Fujita, M., 1989, Urban Economic Theory. Cambridge University Press, Cambridge.

[9] Haavio M. and Kauppi H., 2003. Housing markets and labor mobility. mimeo, Helsinki University.

[10] Holzer, H., 1987. Informal job search and black youth unemployment. American Economic Review 77, 446-452.

[11] Holzer, H., 1988. Search method used by unemployed youth. Journal of Labor Economics $6,1-20$.

[12] Ihlanfeldt, K.R., Sjoquist, D.L., 1990. Job accessibility and racial differences in youth employment rates. American Economic Review 80, 267-276.

[13] Layard, R., Nickell, S., Jackman, R., 1991. Unemployment. Macroeconomic Performance and the labor Market. Oxford University Press, Oxford.

[14] Lucas, R., Prescott, E., 1974. Equilibrium search and unemployment. Journal of Economic Theory $7,188-209$.

[15] Marston, S. T., 1985. Two views of the geographic distribution of unemployment. Quarterly Journal of Economics 100, 57-79.

[16] Moen, E.R., 1997. Competitive search equilibrium. Journal of Political Economy 105, $385-411$.

[17] Mortensen, D.T., Pissarides, C.A., 1999. New developments in models of search in the labor market. In: Card, D., Ashenfelter, O. (Eds.), Handbook of Labor Economics. Elsevier Science B.V, Amsterdam, pp. 2567-2627.

[18] Pissarides, C.A., 2000. Equilibrium Unemployment Theory, 2nd edition, MIT Press, Cambridge, MA.

[19] Rogers, C.L., 1997. Job search and unemployment duration: implications for the spatial mismatch hypothesis. Journal of Urban Economics 42, 109-132.

[20] Seater, J., 1979. Job search and vacancy contacts. American Economic Review 69, 411419.

[21] Smith, T.E., Zenou, Y., 1997. Dual labor markets, urban unemployment, and multicentric cities. Journal of Economic Theory 76, 185-214.

[22] Smith, T.E., Zenou, Y., 2003. Spatial mismatch, search effort and urban spatial structure. Journal of Urban Economics 54, 129-156. 
[23] Stigler, G.J., 1961. The Economics of information. Journal of Political Economy 70, 94-104.

[24] Topa G., 2001. Social interactions, local spillovers and unemployment. Review of Economic Studies 68, 261-295.

[25] Van Ommeren, J., Rietveld, P., Nijkamp, P., 1997. Commuting: in search of jobs and residences. Journal of Urban Economics 42, 402-421.

[26] Wasmer, E., Zenou, Y. 2002. Does city structure affect job search and welfare? Journal of Urban Economics 51, 515-541.

[27] Zax, J., Kain, J.F., 1996. Moving to the suburbs: do relocating companies leave their black employees behind? Journal of Labor Economics 14, 472-493.

[28] Zenou, Y., 2002. How do firms redline workers? Journal of Urban Economics 52, 391-408. 


\section{Appendix 1: Section 2}

\section{Bid rents}

From equations (2) and (3), the bid rents of the unemployed and employed are respectively given by:

$$
\begin{gathered}
\Psi_{u}(d, \bar{U}, \bar{W})=b-\tau_{u} d+p(d) \bar{W}-[r+p(d)] \bar{U} \\
\Psi_{e}(d, \bar{U}, \bar{W})=w-\tau_{e} d+\delta \bar{U}-(r+\delta) \bar{W}
\end{gathered}
$$

By using (34), (35), (7) and replacing them in (8) and (9), we obtain:

$$
\bar{W}-\bar{U}=\frac{w-b-\left(\tau_{e}-\tau_{u}\right) d_{b}}{r+\delta+p\left(d_{b}\right)}
$$

where $w, u, \theta$ will be determined at the labour market equilibrium and $p\left(d_{b}\right)=\left[s_{0}-a(1-u)\right] \theta q(\theta)$.

\section{Wage determination}

The Nash sharing rule leads to:

$$
(\bar{W}-\bar{U})(w)=\frac{\alpha}{1-\alpha} \frac{\gamma}{q(\theta)}
$$

By using (36), we easily obtain (12).

\section{Appendix 2: Section 3}

\section{Bid rents}

The bid rents of the workers are given by:

$$
\begin{aligned}
\Psi_{e}^{m}\left(d, \bar{U}^{m}, \bar{W}^{m}\right) & =w-\tau_{e} d-(r+\delta) \bar{W}^{m}+\delta\left(\bar{U}^{m}-C\right) \\
\Psi_{u}^{m}\left(d, \bar{U}^{m}, \bar{W}^{m}\right) & =b-\tau_{u} d-(r+p) \bar{U}^{m}+p\left(\bar{W}^{m}-C\right) \\
\Psi_{e}^{i}\left(d, U^{i}(d), W^{i}(d)\right) & =w-\tau_{e} d-(r+\delta) W^{i}(d)+\delta U^{i}(d) \\
\Psi_{u}^{i}\left(d, U^{i}(d), W^{i}(d)\right) & =b-\tau_{u} d-(r+p) U^{i}(d)+p W^{i}(d)
\end{aligned}
$$


which implies that one can quantify the rent difference in $d^{\alpha}$ and $d^{\beta}$ as follows, using equations (25) to $(28)$ :

$$
\begin{aligned}
& \Psi_{e}^{m}\left(d^{\alpha}, \bar{U}^{m}, \bar{W}^{m}\right)-\Psi_{e}^{i}\left(d^{\alpha}, U^{i}(d), W^{i}(d)\right)=\delta[C-C]=0 \\
& \Psi_{u}^{m}\left(d^{\beta}, \bar{U}^{m}, \bar{W}^{m}\right)-\Psi_{u}^{i}\left(d^{\beta}, U^{i}(d), W^{i}(d)\right)=p[C-C]=0
\end{aligned}
$$

To determine the slope of the rent in the area between $d^{\alpha}$ and $d^{\beta}$, one can use that $\Psi_{u}^{m}\left(1, \bar{U}^{m}, \bar{W}^{m}\right)=$ 0 at the city edge, from which we obtain a link between $\bar{U}^{m}$ and $\bar{W}^{m}$, namely:

$$
\bar{U}^{m}(r+p)=b-\tau_{u}-p C+p \bar{W}^{m}
$$

while, for all $d$ between $d^{\alpha}$ and $d^{\beta}$, we have

$$
W^{i}(d)-U^{i}(d)=\frac{w-b-\left(\tau_{e}-\tau_{u}\right) d}{r+p+\delta}
$$

We then notice that (23) and (38) implies the first equation below,

$$
\begin{aligned}
& \partial R / \partial d=-r \partial U^{i} / \partial d-\tau_{u}-\frac{p\left(\tau_{e}-\tau_{u}\right)}{r+\delta+p} \\
& \partial R / \partial d=-r \partial W^{i} / \partial d-\tau_{e}+\frac{\delta\left(\tau_{e}-\tau_{u}\right)}{r+\delta+p}
\end{aligned}
$$

while the second is obtained with (24) and (38) but is redundant with the first one.

Finally, note that equations (30) and (31) provide an expression for $\partial U^{i} / \partial d=C\left(d^{\beta}-d^{\alpha}\right)$, which then leads, using the equilibrium value for $d^{\beta}-d^{\alpha}$, to $\frac{2 C^{2}(r+p+\delta)}{\tau_{e}-\tau_{u}}$. Then, using (39), we obtain an expression for $\kappa$, that is

$$
\kappa=\frac{2 r C^{2}(r+p+\delta)}{\tau_{e}-\tau_{u}}+\tau_{u}+\frac{p\left(\tau_{e}-\tau_{u}\right)}{r+\delta+p}
$$

\section{Proof of Lemma 1}

The utility of agents in $\mathcal{I}$ is not constant in space, contrary to the utility of mobile agents. We postulate that it is linear in distance, i.e. $U^{i}(d)$ and $W^{i}(d)$ are linear. Inspection of (23) and (24) suggests that this is the case only if the rent is itself linear. This will be shown ex-post. Thus, the minimum of $U^{i}(d), d \in \mathcal{I}$ is reached either in $d^{\alpha}$ or $d^{\beta}$. In $\mathcal{I}$, we necessarily have $U^{i}(d) \geq \bar{U}^{m}-C$ with equality at the minimum, i.e. $U^{i}\left(d^{\min U}\right)=\bar{U}^{m}-C$. If $d^{\min U}=d^{\beta}$, one would have a contradiction with (26) for strictly positive $C$. Thus, we look for equilibria in which $d^{\min U}=d^{\alpha}$. A similar reasoning applies to the immobile employed. The minimum of utility of employed is thus 
reached at $d^{\beta}$. This implies that, in a non-degenerate equilibrium, (28) and (27) must hold. We proved in this Appendix (see above, equation (38)) that, for all $d$ between $d^{\alpha}$ and $d^{\beta}$, we have

$$
W^{i}(d)-U^{i}(d)=\frac{w-b-\left(\tau_{e}-\tau_{u}\right) d}{r+p+\delta},
$$

i.e., for the immobile workers, the surplus of a job is decreasing with distance from the center. This suggests that indeed, the minimum of the utility of the employed in $\mathcal{I}$ is reached in $d^{\beta}$ and the minimum of the utility of the unemployed is reached in $d^{\alpha}$, so that our postulate is correct. This is formally established at the end of section 3 .

\section{Appendix 3: Section 4}

\section{Proof of Proposition 2}

When workers choose endogenously their effort level, the value of unemployment can be written as:

$$
r U(d)=b-C_{u}(e, d)-R(d)+\theta q(\theta) s(e)\left[\left(\max _{d^{\prime}} W\left(d^{\prime}\right)\right)-U(d)\right]
$$

while the value of employment is still given by (3). The unemployed worker located at a distance $d$ from the employment center chooses $e^{*}$ that maximizes his/her intertemporal utility (41). The first order condition on effort yields:

$$
\theta q(\theta) s^{\prime}\left(e^{*}\right)(\bar{W}-\bar{U})=\partial C_{u}\left(e^{*}, d\right) / \partial e
$$

By totally differentiating (42), we obtain:

$$
\frac{\partial e^{*}}{\partial d}=\frac{\partial^{2} C_{u}\left(e^{*}, d\right) / \partial e \partial d}{\theta q(\theta)(\bar{W}-\bar{U}) s^{\prime \prime}\left(e^{*}\right)-\partial^{2} C_{u}\left(e^{*}, d\right) / \partial^{2} e}<0
$$

and thus

$$
\frac{\partial s}{\partial d}=s^{\prime}(e) \frac{\partial e}{\partial d}<0
$$

\section{An example}

Assume $s(e)=e^{\sigma}$, with $\sigma<1 / 2$ for reasons that will become clear below, and that $C(e, d)=$ $\tau_{u}(e) \cdot d=\left(\tau_{0 u}+\tau_{u} . e\right) d$. By using (42), we easily obtain:

$$
s\left(e^{*}\right)=\left[\frac{\theta q(\theta)(\bar{W}-\bar{U})}{2 \tau_{u} d}\right]^{\frac{\sigma}{1-\sigma}}
$$


or

$$
e^{*}=\left[\frac{\theta q(\theta)(\bar{W}-\bar{U})}{2 \tau_{u} d}\right]^{\frac{1}{1-\sigma}}
$$

One can see that $\sigma<1 / 2$ allows us to integrate $s(e)$ between 0 and any number. We finally obtain:

$$
\bar{s}=\left(\frac{1-2 \sigma}{1-\sigma}\right)\left[\frac{\theta q(\theta)(\bar{W}-\bar{U})}{2 \tau_{u}}\right]^{\frac{\sigma}{1-\sigma}}\left[1-(1-u)^{\frac{1-2 \sigma}{1-\sigma}}\right]
$$

Space has an impact on $\bar{s}$ through the parameter $\tau_{u}$ which is the cross-derivative of the cost function for the unemployed, i.e. $\frac{\partial^{2} C(e, d)}{\partial e \partial d}$. Subsidizing search costs for the unemployed would decrease $\tau_{u}$ and increase aggregate search efficiency. It is also reflected in the term $(1-u)^{\frac{1-2 \sigma}{1-\sigma}}$ which, as before, reflects the average distance between unemployed workers and jobs. 